\title{
Foreign exchange prediction based on indices and commodities price using convolutional neural network
}

\author{
Rian Rassetiadi, Suharjito \\ Computer Science Department, Binus Graduate Program - Master of Computer Science, \\ Bina Nusantara University, Indonesia
}

\begin{tabular}{l}
\hline Article Info \\
\hline Article history: \\
Received Aug 7, 2019 \\
Revised Oct 10, 2019 \\
Accepted Oct 24, 2019 \\
\hline
\end{tabular}

Keywords:

CNN

Commodities

Deep learning

Forex

Indices

\begin{abstract}
The level of accuracy in predicting is the key in conducting forex trading activities in gaining profits. Some predictions are made only by using historical currency data to be predicted, this makes predictions less accurate because they do not consider external influences. This study examines external factors that can influence the results of predictions, by looking for the relationship between the value of indices such as NTFSE and S \& P 500 and the value of commodities such as gold and silver to the prediction process of EUR / USD. Prediction carried out using a deep learning algorithm with the Convolutional Neural Network method uses 4 1dimensional convolution layers with ReLu activation. The data used is the value of Open, High, Low and Close prices on forex, indices and commodities which are combined into one with the close forex value target for the next 1 days. Testing of EUR / USD test data gets MSE results of 0.00009984 . While the results of testing of the combined test data between EUR / USD, indices and commodities producing MSE vary between 0.0000589 to 0.000137 where the best combination is a combination of FTSE 100 and Natural Gas values. So, it can be concluded that other factors included in predicting have an influence on the results obtained.
\end{abstract}

Copyright $@ 2020$ Institute of Advanced Engineering and Science. All rights reserved.

\section{Corresponding Author:}

Suharjito,

Computer Science Department,

Binus Graduate Program - Master of Computer Science,

Bina Nusantara University,

Jakarta, Indonesia 11480.

Email: suharjito@binus.edu

\section{INTRODUCTION}

A currency market is a market that has a significant effect on world financial flows, this is because the currency market is a place where people sell to buy the value of a currency against other currencies in the world [1]. The market is also the most active financial market, because the number of transactions is very large with continuous operations and trading starts from 22:00 to 17:00 GMT Sunday to 22:00 GMT Friday (New York) [2]. The size of a currency market is up to thirty times the turnover of the stock market owned by the United States where there is approximately 3 trillion dollars in flow of funds every day [3]. Activities in buying and selling currencies are often also called forex trading. The movement of currency exchange rates in general is very difficult to predict the direction of its speed. Not a few people who buy and sell foreign currency and benefit from the transaction [4]. Moving on in the business of buying and selling currencies has a considerable opportunity to make a profit, but the losses that can be obtained are also no less great if the decisions taken in buying and selling are not carefully considered.

The level of accuracy in predicting is key in conducting forex trading activities [5]. Some predictions are made only by using historical currency data to be predicted, this makes predictions less accurate because they do not consider external influences [6]. The method approach in predicting currency 
exchange rate movements is two ways, namely using fundamental analysis and technical analysis [7]. The Random Walk concludes that the movement of a price cannot be predicted accurately using historical value [8]. However, advances in artificial intelligence and the growth of available data have made it possible to estimate price movement behavior with better performance than random processes $[9,10]$. Therefore this research is conducted by using technical analysis by examining external factors that can influence the outcome of predictions, by looking for the linkages between stock values such as NTFSE and S \& P 500 and commodity values such as gold and silver towards the EUR / USD prediction process using deep learning algorithms with CNN method [11].

In previous studies, it has shown the performance of CNN applied to predictions of forex movements, namely against the EUR / USD, GBP / USD and USD / JPY pairs. The method used is CNN with 2-dimensional convolution using the LeNet-5 architecture. The data that becomes the input is the result of the transformation of the price taking every hour for 24 days, so that the matrix is $24 \times 24$. In this study using adagard as the optimization method. The results obtained from predicting one day ahead in the study produced MSE of 0.000162 [2]. The Random Walk concludes that the movement of a price cannot be predicted accurately using historical value [8]. There is research conducted by predicting USD / CHF with backpropagation neural network design which results in a trend improvement accuracy of $74 \%$, the study uses USD / CHF data as training data and input data. The method used is backpropagation using a combination of 4 input neurons, 3 hidden layers with the number of neurons (8: 4: 2) and output that is the direction of trend change described using 1 output neuron [1]. However, advances in artificial intelligence and the growth of available data have made it possible to estimate price movement behavior with better performance than random processes [9]. Deep Learning began to be used both CNN and LSTM for the sake of predictions, comparisons have been made between MLP, CNN and RNN with CNN results having a higher level of accuracy than other methods [7]. All deep learning methods in the study have better results compared to methods that are not deep learning, because deep learning can take more features in the learning process. As has been done for the prediction of the S \& P 500 joint stock index with an accuracy of 62\% [7]. Prediction is a unique material to study because its movements are so unpredictable, therefore factors that can influence the rate of predictions are included. Including other currency pairs to predict a currency using backpropagation can affect the prediction results, namely the prediction of EUR / GBP by presenting GBP / USD as a factor and the backpropagation method used [12]. Using the deep learning method using CNN has also been done on the prediction of USD / GBP by presenting external factors, namely oil and gold values and proven to influence predictive accuracy. The prediction is done by using the CNN method by using 4 hidden layers [13].

After doing some literature studies it has been found that making predictions by paying attention to the factors that influence will produce more precise predictive values and the problem is predicting without any factor included will be less accurate than predicting with external factor. Thus, this research will be carried out by considering the factors that influence the movement of the exchange rate of EUR / USD by combining several values of stocks, forex and commodity using the deep learning method. Adam's method will be used to optimize gradient descent in the learning process, because the Adam optimization method is a good optimization method and is suitable for use in machine learning optimization [14].

\section{PROPOSED METHOD}

CNN is an interesting technique for high-dimensional data, such as images and time series data. CNN has been widely applied for feature selection and prediction of price movements [15]. In the convolutional neural network, not all hidden neurons are connected to each other [16, 17]. The training process and testing carried out in this study used the CNN method. The hyperparameter used is 4 layers, and both of them are 1-dimensional convolutions.

The proposed method can be seen in Figure 1. The first hidden layer is conv1D with a kernel size of $5 \times 1$ and 24 filters. The output of the first hidden layer is $16 \times 1 \times 24$. The second hidden layer is conv1D with a kernel size of $5 \times 1$ and 48 number of filters. The output of the second hidden layer is $12 \times 1 \times 48$. The third hidden layer is conv1D with a kernel size of $5 \times 1$ and 48 number of filters. The output of the third hidden layer is $8 \times 1 \times 48$. The last hidden layer is the fourth hidden layer is conv1D with a kernel size of $5 \mathrm{x}$ 1 and 24 number of filters. The output of the fourth hidden layer is $6 \times 1 \times 24$.

The last layer is the output layer. At the output layer there is one $1 \times 1$ matrix with 12 filters. At the output layer the ReLU activation function is applied. 


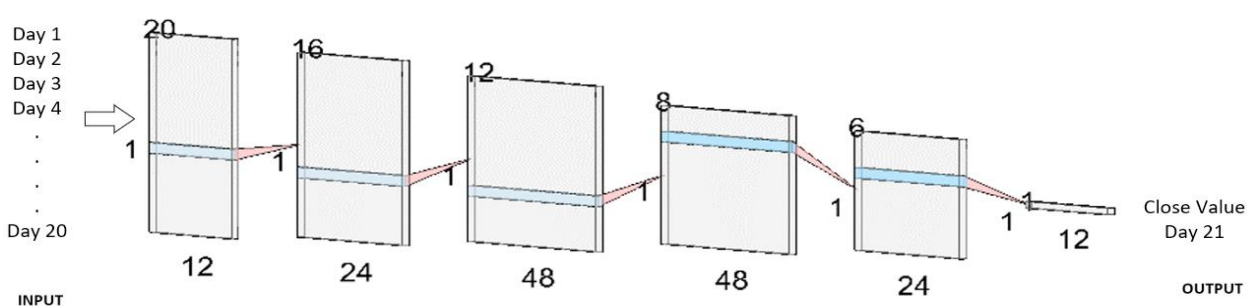

Figure 1. Proposed CNN method

\section{RESEARCH METHOD}

The method used in this study is to use a deep learning algorithm with the CNN method. Open High Low Close (OHLC) values are used to make predictions. The research method is to combine the EUR / USD OHLC price movement values with the OHLC stock price and commodities to produce a prediction of EUR / USD values at $t+1$, can be seen in Figure 2. In the first step eur-usd data, indices and commodity taken. After the data is collected, the preprocessing is done on each data, the preprocessing that is done includes the disposal of dirty data, normalization and removal of unnecessary attributes such as the volume contained in each data. After preprocessing is complete, the three data are combined into one. The training process is carried out using the CNN method. Besides the CNN method can be used for processing data in the form of images can also be used for data in the form of datasets, the dataset used will be taken as many as 20 days. The combination of OHLC between EUR / USD, stocks and commodities will produce 12 values.

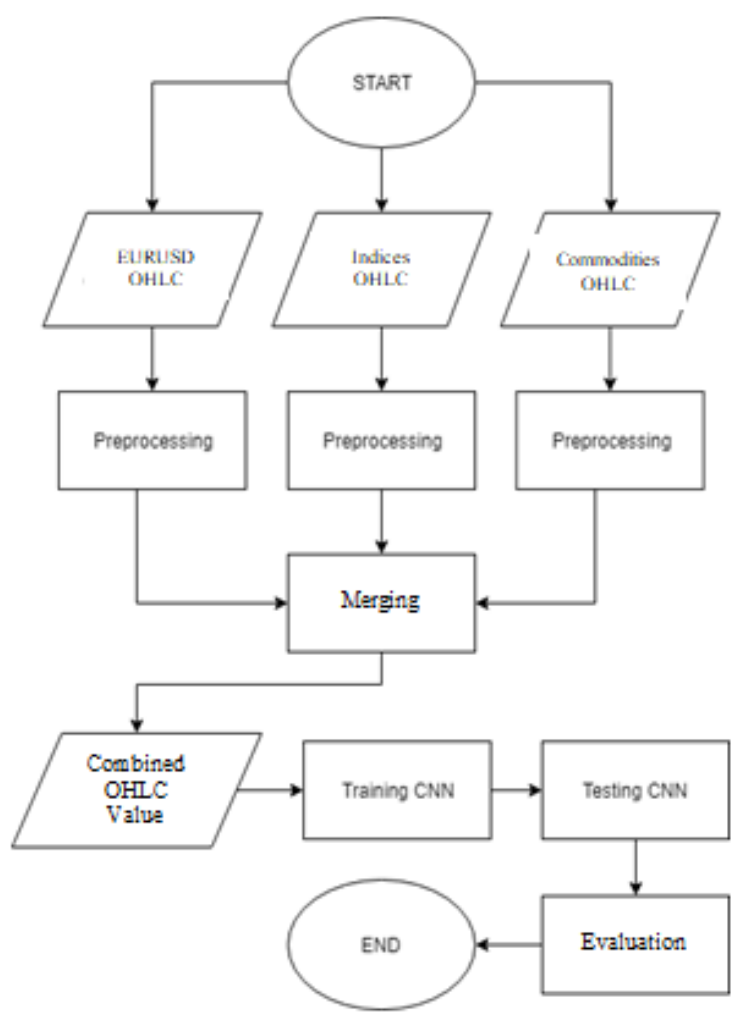

Figure 2. Research method

\subsection{Preprocessing}

Preprocessing in this study is used to equalize all data values from each price data. Preprocessing is done with each data using (1). 
Where:

$$
\text { normalized }(x)=\frac{x-\text { minValue }}{\text { maxValue }- \text { minValue }}
$$

$x=$ Value to be normalized

minValue $=$ Lowest value

maxValue $=$ Highest value

\subsection{Data Input}

Data input is formed from the merger of three prices, namely forex prices, indices and commodities, where each has an open, high, low and close (OHLC) value. Segmentation is done not too long to avoid the problem of lack of memory storage during the training process [18]. In Figure 3 is an example of the input data formed after the merger, the data is sorted by OHLC forex and OHLC indices and OHLC commodities. After the merging process is complete then one training data and one test data are made into 20 working days.

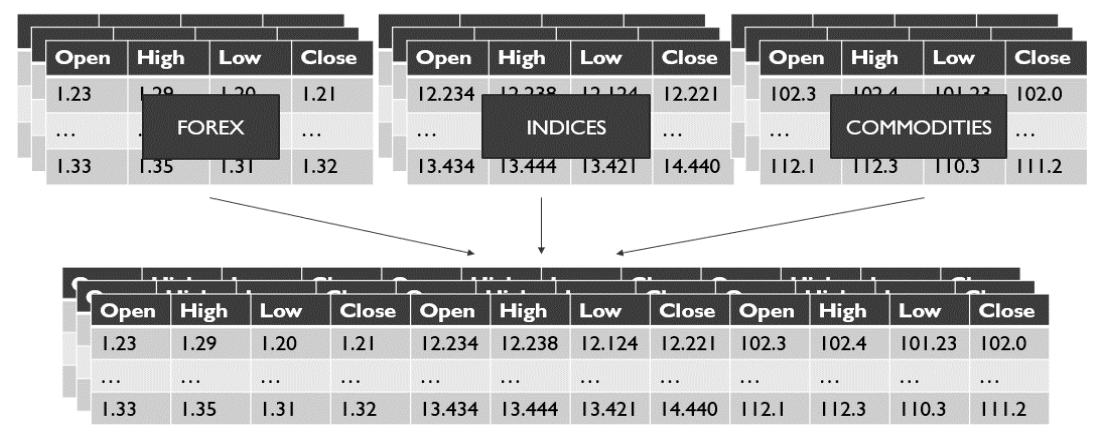

Figure 3. Examples of segmentation of EUR / USD, GOLD and FTSE 100 as input data

\subsection{Evaluation}

The evaluation results will be measured by calculating the Mean Squared Error (MSE) [19] on each test. The MSE formula can be seen in (2).

$$
M S E=\frac{1}{n} \sum_{i=1}^{n}\left(Y_{i}-Y_{i}\right)^{2}
$$

Evaluation will be carried out using as much as $20 \%$ of all data, namely data from 2016 to 2018 . Evaluation is done by testing the model that has been made by measuring the MSE value of each test data tested and compared with the MSE value of prediction testing results regardless of indices and commodities [20].

\section{RESULTS AND DISCUSSION \\ 4.1. Data Description}

The data used in this study was taken from www.investing.com. Data is taken directly by downloading and data in the form of CSV. Data was taken from January 2000 to October 2018. Forex data taken is EUR-USD, data for indices taken are DAX, Dow, FTSE 100, Nasdaq and S \& P 500, while data for commodities taken are Brent oil, copper, gold, natural gas and silver. The data obtained contains the values of date, close, open, high, low, vol and change. Because the data needed is only date, close, open, high and low, vol and change are not included or deleted from the dataset.

Data to be used must be pairs between forex, commodities and indices. So that data selection must be done by removing data that does not have a partner on a certain date. There are several days in some data that do not have value so cleaning must be done by deleting the partner data. For example, on May 10, 2017 the data of eur-usd and dax has a value, while for silver at that time it has no value, then the data on May 10, 2017 is deleted. The results of cleaning result only from January 2007 to October 2018, the data can be used and paired together. The data obtained is divided into two, namely for test data and training data with a ratio of $80 \%$ for training data and $20 \%$ for test data. 


\subsection{Experimental Setup}

Experiment is done using the python programming language as a data processing tool. The first thing to do is determine the combination of forex, indices and commodities. In Table 1 is a combination of all possibilities and formed 25 pairs of factors that are ready for training and testing.

The following are eur-usd, gold and FTSE 100 data from the results of retrieval from www.investing.com that have not been merged. Each data has a date, price, open, high, low and Change\% columns. For data commodities and indices there is a Volume column. In Table 2 is an example of historical data eur-usd which is still not entering the merging stage, there are still some unnecessary attributes such as Change $\%$.

Table 1. Forex, Indices dan Commodities

\begin{tabular}{cccc}
\multicolumn{4}{c}{ Combination } \\
\hline No & Forex & Commodities & Indices \\
\hline 1 & EUR-USD & Copper & DAX \\
2 & EUR-USD & Copper & DOW \\
3 & EUR-USD & Copper & FTSE 100 \\
4 & EUR-USD & Copper & NASDAQ \\
5 & EUR-USD & Copper & S\&P 500 \\
6 & EUR-USD & Natural Gas & DAX \\
7 & EUR-USD & Natural Gas & DOW \\
8 & EUR-USD & Natural Gas & FTSE 100 \\
9 & EUR-USD & Natural Gas & NASDAQ \\
10 & EUR-USD & Natural Gas & S\&P 500 \\
11 & EUR-USD & Gold & DAX \\
12 & EUR-USD & Gold & DOW \\
13 & EUR-USD & Gold & FTSE 100 \\
14 & EUR-USD & Gold & NASDAQ \\
15 & EUR-USD & Gold & S\&P 500 \\
16 & EUR-USD & Oil & DAX \\
17 & EUR-USD & Oil & DOW \\
18 & EUR-USD & Oil & FTSE 100 \\
19 & EUR-USD & Oil & NASDAQ \\
20 & EUR-USD & Oil & S\&P 500 \\
21 & EUR-USD & Silver & DAX \\
22 & EUR-USD & Silver & DOW \\
23 & EUR-USD & Silver & FTSE 100 \\
24 & EUR-USD & Silver & NASDAQ \\
25 & EUR-USD & Silver & S\&P 500 \\
\hline & & &
\end{tabular}

Table 2. Example EUR-USD Historical Data

\begin{tabular}{cccccc}
\hline Date & Price & Open & High & Low & Change \% \\
\hline 4-Oct-18 & 1.1515 & 1.1478 & 1.1543 & 1.1464 & $0.32 \%$ \\
3-Oct-18 & 1.1478 & 1.1548 & 1.1595 & 1.1465 & $-0.61 \%$ \\
2-Oct-18 & 1.1548 & 1.1578 & 1.1582 & 1.1506 & $-0.26 \%$ \\
1-Oct-18 & 1.1578 & 1.1609 & 1.163 & 1.1563 & $-0.27 \%$ \\
28-Sep-18 & 1.1609 & 1.1641 & 1.1652 & 1.1567 & $-0.27 \%$ \\
27-Sep-18 & 1.1641 & 1.174 & 1.1759 & 1.1637 & $-0.84 \%$ \\
\hline
\end{tabular}

In Table 3 is an example of historical data for commodities that are still not entering the merger stage, there are still some unnecessary attributes such as Change $\%$ and Volume. In Table 4 is an example of historical data for indices that are still not entering the merging stage, there are still some unnecessary attributes such as Change\% and Volume.

Table 3 Example Gold Historical Data

\begin{tabular}{ccccccc}
\hline Date & Price & Open & High & Low & Vol. & Change \% \\
\hline 4-Oct-18 & 1201.6 & 1201.4 & 1210.7 & 1199.6 & $290.84 \mathrm{~K}$ & $-0.11 \%$ \\
3-Oct-18 & 1202.9 & 1207 & 1212.3 & 1200.4 & $255.07 \mathrm{~K}$ & $-0.34 \%$ \\
2-Oct-18 & 1207 & 1192.7 & 1212.3 & 1192.2 & $365.32 \mathrm{~K}$ & $1.28 \%$ \\
1-Oct-18 & 1191.7 & 1196.1 & 1196.5 & 1188.1 & $220.51 \mathrm{~K}$ & $0.02 \%$ \\
28-Sep-18 & 1191.5 & 1181.7 & 1191.9 & 1180 & $307.82 \mathrm{~K}$ & $0.78 \%$ \\
27-Sep-18 & 1182.3 & 1194.6 & 1197.3 & 1180.5 & $351.05 \mathrm{~K}$ & $-1.35 \%$ \\
\hline
\end{tabular}

Table 4. Example FTSE 100 Historical Data

\begin{tabular}{ccccccc}
\hline Date & Price & Open & High & Low & Vol. & Change \% \\
\hline 4-Oct-18 & 7418.34 & 7510.28 & 7510.28 & 7411.31 & $826.76 \mathrm{M}$ & $-1.22 \%$ \\
3-Oct-18 & 7510.28 & 7474.55 & 7524.06 & 7474.55 & $849.12 \mathrm{M}$ & $0.48 \%$ \\
2-Oct-18 & 7474.55 & 7495.67 & 7495.67 & 7444.8 & $843.12 \mathrm{M}$ & $-0.28 \%$ \\
1-Oct-18 & 7495.67 & 7510.2 & 7526.2 & 7466.67 & $746.18 \mathrm{M}$ & $-0.19 \%$ \\
28-Sep-18 & 7510.2 & 7545.44 & 7548.36 & 7474.67 & $943.92 \mathrm{M}$ & $-0.47 \%$ \\
27-Sep-18 & 7545.44 & 7511.49 & 7552.02 & 7490.94 & $666.22 \mathrm{M}$ & $0.45 \%$ \\
\hline
\end{tabular}


In Table 5 is an example of the merged eurusd-gold-ftse data. Naming is changed to close, open, high, low for eurusd data. cLose, cOpen, cHigh, cLow for commodity data and iClose, iOpen, iHigh, iLow for data indices. While the date column is changed to the timestamp format. As the target or value that will be predicted is the value close from eur-usd.

The prediction process is done through the training phase through the CNN process with 500 epochs. The weight obtained is stored every epoch on the storage media so that weights can be used for testing.

Table 5. EUR-USD-Gold-FTSE

\begin{tabular}{|c|c|c|c|c|c|c|c|c|c|c|c|c|}
\hline Close & Open & High & Low & cClose & cOpen & cHigh & cLow & iClose & iOpen & iHigh & iLow & Timestamp \\
\hline 1.3085 & 1.3167 & 1.3182 & 1.3074 & 623.9 & 624.5 & 624.5 & 624.5 & 6287 & 6319 & 6319 & 6261 & 1167843600 \\
\hline 1.3003 & 1.3085 & 1.3106 & 1.298 & 604.9 & 623.7 & 623.7 & 617.3 & 6220.1 & 6287 & 6287 & 6220.1 & 1167930000 \\
\hline 1.302 & 1.3013 & 1.3037 & 1.2973 & 607.5 & 607.5 & 607.5 & 607.5 & 6194.2 & 6220.1 & 6246 & 6187 & 1168189200 \\
\hline 1.2997 & 1.3023 & 1.3054 & 1.2985 & 613.1 & 607 & 607 & 607 & 6196.1 & 6194.2 & 6218.5 & 6190.4 & 1168275600 \\
\hline 1.2934 & 1.3002 & 1.3008 & 1.2931 & 611.6 & 611.6 & 611.6 & 611.6 & 6160.7 & 6196.1 & 6196.1 & 6142 & 1168362000 \\
\hline 1.2893 & 1.2937 & 1.3016 & 1.2881 & 612.4 & 612.4 & 612.4 & 612.4 & 6230.1 & 6160.7 & 6233.1 & 6130.2 & 1168448400 \\
\hline 1.2917 & 1.2891 & 1.2946 & 1.2866 & 625.5 & 616.2 & 616.2 & 616.2 & 6239 & 6230.1 & 6247.6 & 6204.3 & 1168534800 \\
\hline$\ldots$ & $\ldots$ & $\ldots$ & $\ldots$ & 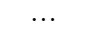 & 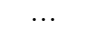 & - & $\ldots$ & $\ldots$ & 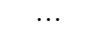 & -2 & $\cdots$ & $\ldots$ \\
\hline 1.1548 & 1.1578 & 1.1582 & 1.1506 & 1207 & 1192.7 & 1212.3 & 1192.2 & 7474.55 & 7495.67 & 7495.67 & 7444.8 & 1538413200 \\
\hline 1.1478 & 1.1548 & 1.1595 & 1.1465 & 1202.9 & 1207 & 1212.3 & 1200.4 & 7510.28 & 7474.55 & 7524.06 & 7474.55 & 1538499600 \\
\hline 1.1515 & 1.1478 & 1.1543 & 1.1464 & 1201.6 & 1201.4 & 1210.7 & 1199.6 & 7418.34 & 7510.28 & 7510.28 & 7411.31 & 1538586000 \\
\hline
\end{tabular}

\subsection{Result}

Testing is done by using $20 \%$ of the data that has been obtained, namely data from 2016 to 2018 . The target data is the close value of the EUR-USD currency. Following the blue point in Figure 4 is the movement of the value of MSE repairs during the training period. Training is conducted with 500 epochs.

The test results for each combination have varying MSE values. The results obtained are compiled based on factors namely indices and commodities. Can be seen in Table 6 that the results of 25 experiments combining factors against eur-usd produce the best value 0.0000589, which is the incorporation of the Natural gas factor with the FTSE100. While predictions using EUR-USD alone without using the indices and commodities factors get the MSE yield of 0.000099 .

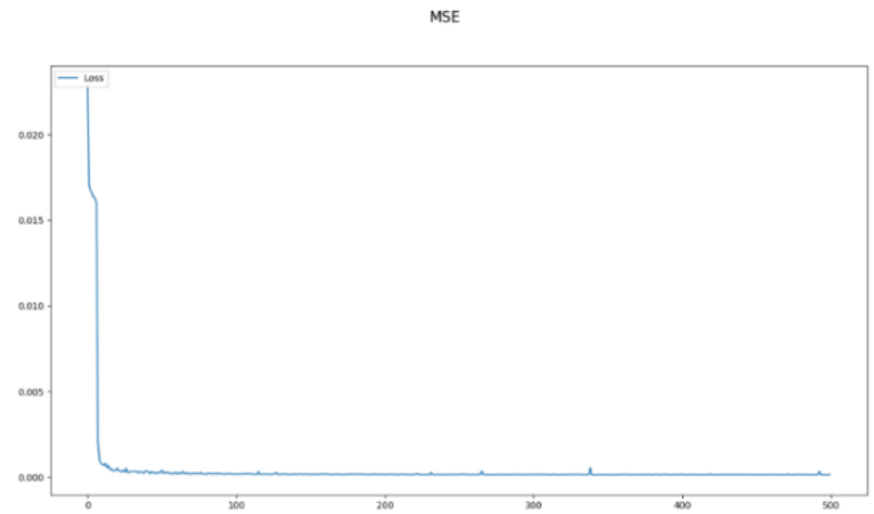

Figure 4. MSE training

Table 6. Result MSE Combination EUR-USD with Commodities and Indices

\begin{tabular}{cccccc}
\hline & Dax & Dow 30 & FTSE 100 & Nasdaq 100 & S\&P 500 \\
\hline Copper & 0.0000887 & 0.0000811 & 0.0000948 & 0.0000974 & 0.0000739 \\
Natural Gas & 0.0000797 & 0.0000641 & 0.0000589 & 0.0000778 & 0.0000824 \\
Gold & 0.000109 & 0.000101 & 0.0000802 & 0.0000775 & 0.0000962 \\
Brent Oil & 0.0000855 & 0.0000686 & 0.000137 & 0.0000848 & 0.000105 \\
Silver & 0.0000824 & 0.0000667 & 0.0000947 & 0.0000728 & 0.0000605
\end{tabular}

In Figure 5 is an example of predictions made from 2016 to 2018 with the factors used namely natural gas and FTSE100. The blue line illustrates the actual movement of EUR-USD, while the red dot is a

Foreign exchange prediction based on indices and commodities price using... (Rian Rassetiadi) 
prediction produced by the system. The results show that the prediction results can be used as a tool in determining price movements.

In this study also shows that the role of indices and commodities affects the results of predictions. Table 7 shows the results of comparisons obtained from combining index and commodity as factors in input data with results obtained without using other factors in input data.

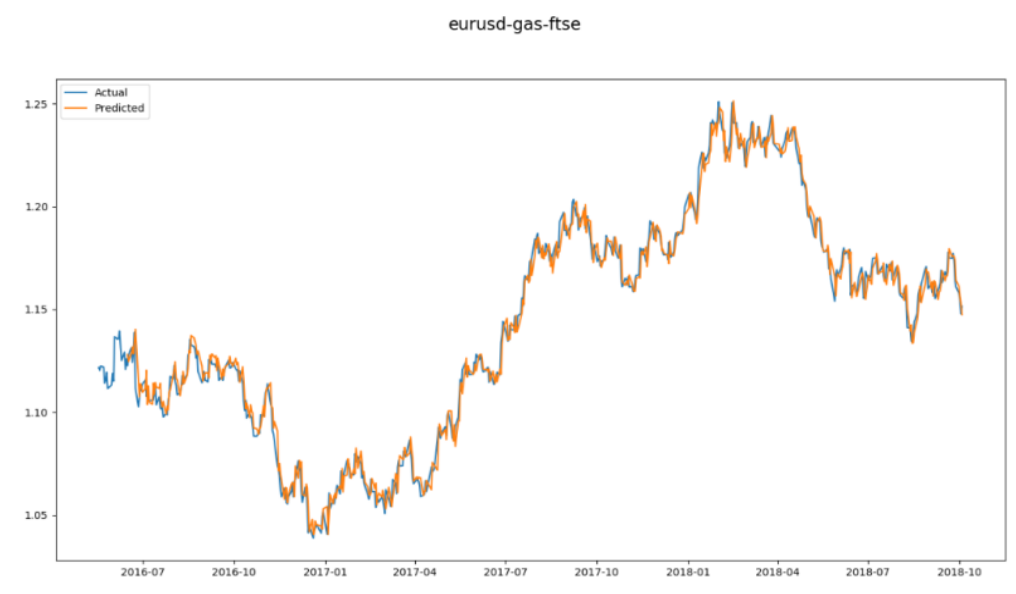

Figure 5. EUR-USD, Natural Gas, FTSE 100

\section{CONCLUSION AND FEATURE WORK}

In this study it can be concluded that the prediction of EUR-USD forex by considering other factors, namely index and commodity values can produce variable MSE values. This can prove other factors besides the currency that will be predicted can affect the value of the prediction itself. In this study the combination of Natural Gas and FTSE 100 can produce a best MSE value of 0.000058 , when compared to other combinations and predictions without including other factors which are equal to 0.000162 and 0.000099 . Thus predictions using this method can improve the value of verification in predicting and increasing accuracy in predicting. CNN with 1-dimensional convolution still produces less accurate predictive values. There are still many shortcomings in research, especially on the CNN architecture, such as using 2 or 3dimensional convolution and presenting optimization in the selection of features to produce predictions to be more precise.

\section{REFERENCES}

[1] D. Khoonmirzaie, S. Rasouli and E. Farrokhi, "Designing Perceptron Three-Layered Neural Network for Predicting Dollar-Franc Currency Pair in International Exchange Market," Journal of mathematics and computer science, vol. 8, no. 2, pp. 98 - 104, 2014.

[2] L. Chen, H. Weiyan and L. Deyin, "Foreign Exchange Rates Forecasting with Convolutional Neural Network," Neural Processing Letters, vol. 46, no. 3, pp. 1095-1119, 2017.

[3] P. Czekalski, M. Niezabitowski and R. Styblinski, "ANN for FOREX Forecasting and Trading," in International Conference on Control Systems and Science, 2015.

[4] S. Galeshchuk and S. ukherjee, "Deep networks for predicting direction of change in foreign exchange rates," Intelligent Systems in Accounting, Finance and Management, vol. 24, no. 4, pp. 100-110, 2017. 
[5] H. Gunduz, Y. Yaslan and Z. Cataltepe, "Intraday prediction of Borsa Istanbul using convolutional neural networks and feature correlations," Knowledge-Based Systems, vol. 137, pp. 138--148, 2017.

[6] Y.-C. Tsai, J.-H. Chen and J.-J. Wang, "Predict Forex Trend via Convolutional Neural Networks," Journal of Intelligent Systems, 2018.

[7] L. D. Persio and O. Honchar, "Artificial neural networks approach to the forecast of stock market price movements," International Journal of Economics and Management Systems, vol. 1, no. 2367-8925, pp. 158-162, 2016.

[8] R. Singh and S. Srivastava, "Stock prediction using deep learning," Multimedia Tools and Applications, vol. 76, no. 18, pp. 18569-18584, 2017.

[9] M. R. Vargas, B. S. De Lima and A. G. Evsukoff, "Deep learning for stock market prediction from financial news articles," in International Conference on Computational Intelligence and Virtual Environments for Measurement Systems and Applications (CIVEMSA), Brazil , 2017.

[10] V. A. Ahnaf and Suharjito, "Financial Revenue Prediction Model in Mining Industry Using Deep Learning," ICIC Express Letters, vol. 13, no. 11, p. 987-993, 2019.

[11] N. A. Rahmad, N. A. J. Sufri, N. H. Muzamil and M. A. As'ari, "Badminton player detection using faster region convolutional neural network," Indonesian Journal of Electrical Engineering and Computer Science, vol. 14, no. 3, pp. 1330-1335., 2019.

[12] I. Ramadhani, Jondri and R. Rismala, "Prediction of Multi Currency Exchange Rates Using Correlation Analysis and Backpropagation," International Conference on ICT For Smart Society, pp. 63-68, 2016.

[13] J. Korczak and M. Hernes, "Deep Learning for Financial Time Series Forecasting in A-Trader System," Computer Science and Information Systems, vol. 11, no. 2300-5963, p. 905-912, 2017.

[14] D. P. Kingma and J. Ba., "Adam: A method for stochastic optimization."," arXiv preprint arXiv, p. 1412.6980, 2014.

[15] E. Hoseinzade and S. Haratizadeh, "CNNPred: CNN-based stock market prediction using several data sources," arXiv preprint arXiv:1810.08923, 2018.

[16] M. Längkvist, L. Karlsson and A. Loutfi, "A Review of Unsupervised Feature Learning and Deep Learning for Time-Series Modeling," Pattern Recognition Letters, vol. 42, pp. 11-24, 2014.

[17] Suharjito, H. Gunawan, N. Thiracitta and A. Nugroho, "Sign Language Recognition Using Modified Convolutional Neural Network Model," in 2018 Indonesian Association for Pattern Recognition International Conference (INAPR), 2018.

[18] Y.-C. Tsai, J.-H. Chen and J.-J. Wang, "Financial Time-series Data Analysis using Deep Convolutional Neural Networks," in International Conference on Cloud Computing and Big Data, 2016.

[19] M. Alkaff, H. Khatimi, W. Puspita and Y. Sari, "Modelling and predicting wetland rice production using support vector regression," Telkomnika, vol. 17, no. 2, 2019.

[20] Y. Abdillah and Suharjito, "Failure prediction of e-banking application system using adaptive neuro fuzzy inference system (ANFIS)," International Journal of Electrical and Computer Engineering, vol. 9, no. 1, p. 667, 2019.

\section{BIOGRAPHIES OF AUTHORS}

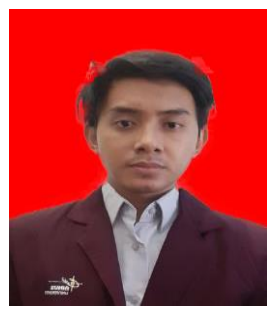

Rian Rassetiadi was born on 11 February 1993 in Bandung, West Java, Indonesia. He graduated in 2015 in Computer Science from UNJANI Cimahi. His research interests include price movement forecast and artificial neural network. Currently Faculty Member at School of Computer Science Binus Graduate Program in Bina Nusantara University.

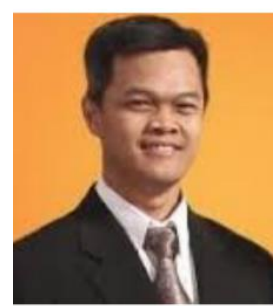

Suharjito, is Head of Magister in Information Technology Department of Binus Graduate Program in Bina Nusantara University. He received under graduated degree in mathematics from The Faculty of Mathematics and Natural Science in Gadjah Mada University, Yogyakarta, Indonesia in 1994. He received master's degree in information technology engineering from Sepuluh November Institute of Technology, Surabaya, Indonesia in 2000. He received the $\mathrm{PhD}$ degree in system engineering from the Bogor Agricultural University (IPB), Bogor, Indonesia in 2011. His research interests are intelligent system, Fuzzy system, image processing and software engineering. 